\title{
Generation of New Eco-friendly Composite Materials via the Integration of Ecodesign Coefficients
}

\author{
Brahim Attaf \\ Expert in Composite Materials \& Structures
}

France

\section{Introduction}

Thanks to their excellent formability, their mass-saving advantage, their high stiffness-todensity and strength-to-density ratios, i.e., $E / \rho$ and $\sigma / \rho$ and the greater freedom to tailor these high properties in the desired orientation and position, fibre-reinforced polymer (FRP) composites are used in many fields of engineering, from architectural structures, ship superstructures, automobiles, bridge decks, machine parts, dams and reservoirs, to the high technology of the modern aerospace industries (Attaf \& Hollaway, 1990a,b). Furthermore, these lightweight materials have some precise objectives, which cannot be reached with some other conventional materials. These attractive advantages coupled with economic design have lead to open up many opportunities to design and manufacture new composite materials and structures for future applications. However, these materials have to satisfy ecodesign requirements, which are based on new standards for designing environmentallyfriendly composite products. Within this context, the industrial designers, manufacturers and suppliers who work in the field of composites are having to factor in the impacts of their products on the environment and find new feasible alternatives. Typically, these alternatives are based on a set of equations, called "ecodesign function" (Attaf, 2007). This function must guarantee quality assurance, health protection and environmental preservation all at the same time, making it necessary to come up with ecodesign strategies that include cleaner production, so as to be in compliance with new regulations and still make the product more competitive in the worldwide market.

With this approach as an objective, codes and standards for future composite materials and structures should integrate, at each stage of the designing process, three balanced key criteria characterised mainly by quality assurance $(Q$ for short), health protection $(H$ for short) and environmental preservation ( $E$ for short). To achieve these requirements, we have defined and developed new criteria in the form of coefficients. Taking into account the previously specified ecological considerations, these coefficients are now called "ecocoefficients". To assess these eco-coefficients, probability approach (Attaf, 2009) and optimisation procedures based on additive colours technique are undertaken in this analysis. And once these eco-coefficients are determined and approved by ecodesign standards, they can then be integrated into the formulations of design and analysis, in characterisation tests; they can also be implemented into future finite-element computer 
programs, etc. In addition, by simply undertaking a comparison of eco-results with classical ones, which do not take into account eco-coefficients, designers and analysts can make better use of ecodesign aspects to assess environmental and health performances.

The aim of this investigation is regarded as: (i) a stimulation for innovation, sustainability and research activities within the field of ecodesign of composite materials and structures; and (ii) an encouragement for designers and engineers involved with high-technology composite materials to have more motivation towards the integration of $Q-H-E$ aspects into the development process of FRP products.

\section{Ecodesign of composite materials and structures}

\subsection{Position of ecodesign approach within sustainable development concept}

According to most scientific results related to the protection of biodiversity, global warming and climate change may have severe effects on human health and the environment. To improve the well-being and living conditions of present and future generations, it is important that the negative impacts generated from human and industrial activities should be seriously considered in all design phases of a new development. To this end, the sustainable development concept is a strong key issue aiming to achieve the previously discussed objective, which is obviously based on three main criteria or "pillars"; these are: (i) environmental sustainability, (ii) economic sustainability and (iii) social sustainability. As the concept of sustainable design or simply ecodesign is inseparable from the sustainable development concept, it is therefore an undissociable part of it, where quality assurance, health protection and environmental preservation aspects are considered to be important branches belonging to the previously described main pillars. Figure 1 illustrates the specific ecodesign situation in relation to the sustainable development concept.

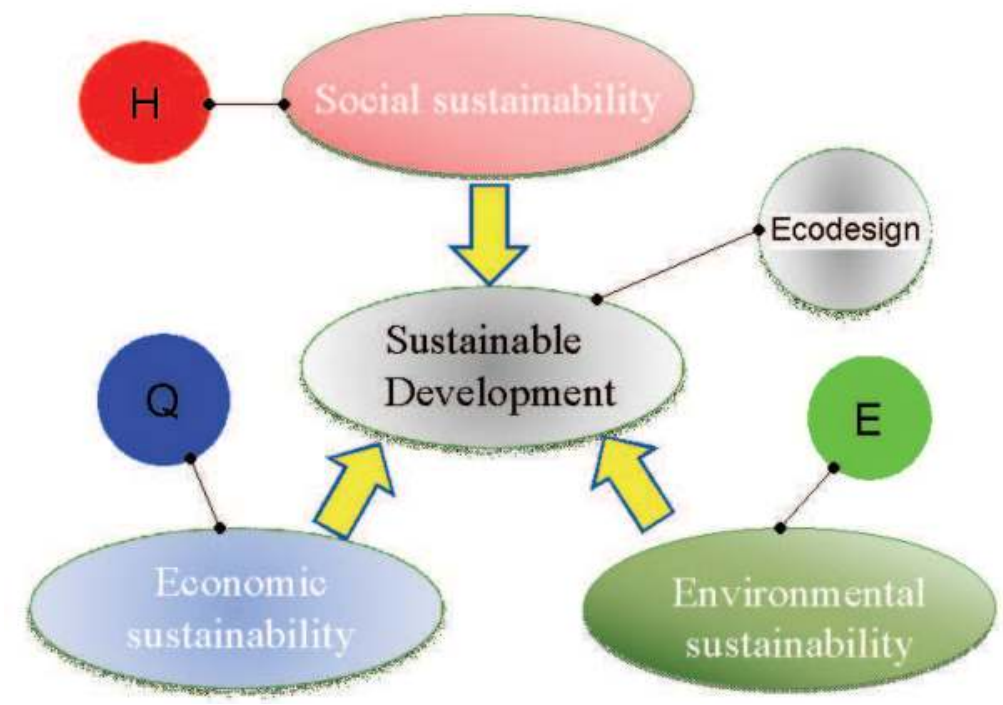

Fig. 1. Ecodesign approach vs. sustainable development concept 


\subsection{Evolution of the interaction between Q-H-E aspects}

The main condition of ecodesign can be reached when the interaction between $Q, H, E$ aspects yields a common area of intersection between these aspects, i.e., $Q \cap H \cap E$. The original diagram (Figure 2a) shows health, quality and environment as three separate aspects that operate independently from each other. Joining the $Q, H, E$ aspects gives rise to a new diagram fulfilling the ecodesign condition (Attaf, 2007) that is characterised by the subset $\ddot{\vec{F}}$ resulting from this intersection (Figure 2 b). The three dots $(\therefore)$ above the character $F$ are only a brief description of the diagram illustrated in Figure 2a, showing interaction between health, quality and environment aspects. In other terms, the three dots represent the three pillars that characterise the basic elements of the sustainable development concept, as discussed above (Figure 1).

Depending on the resultant area of interaction, an optimisation process can be applied to subset $\ddot{F}$ to maximise this area. If optimisation is highly improved ( $\dot{\vec{F}}=\ddot{\mathscr{F}}_{\max }$ ), then the future diagram illustrated in Figure $1 \mathrm{c}$ is achieved and the searched objective is reached, however.

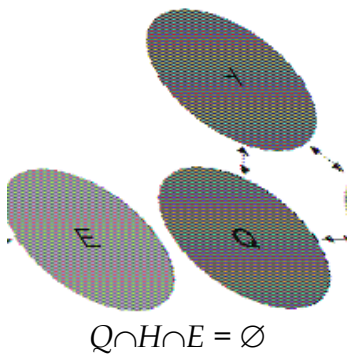

(a) original diagram

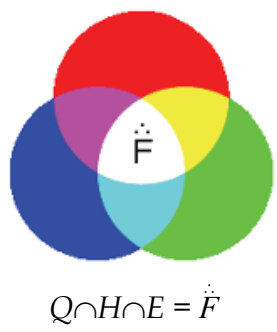

(b) actual diagram

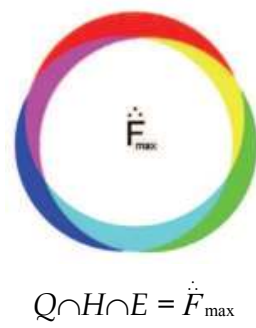

(c) future diagram

Fig. 2. Evolution of the interaction between $Q, H$, and $E$ aspects

\subsection{Ecodesign model and sample space}

As it was shown in Figure 2c, the interaction between the three $Q-H-E$ aspects yields the apparition of a certain number of events, which are illustrated in Figure 3. Each event is assumed to accomplish one or several functions that are defined by the following subsets (Attaf, 2007)

- Subset $A(\square)$ : characterized by an assured quality, a non-protected health and a nonpreserved environment.

- Subset $B(\square)$ : characterized by an assured quality, a protected health and a nonpreserved environment.

- Subset $C(\square)$ : characterized by a non-assured quality, a protected health and a nonpreserved environment.

- Subset $D(\square)$ : characterized by a non-assured quality, a protected health and a preserved environment.

- Subset $S(\square)$ : characterized by an assured quality, a non-protected health and a preserved environment.

- Subset $\ddot{F}(\square)$ : characterized by an assured quality, a protected health and a preserved environment. 
- Subset $G(\square)$ : characterized by an assured quality, a non-protected health and a preserved environment.

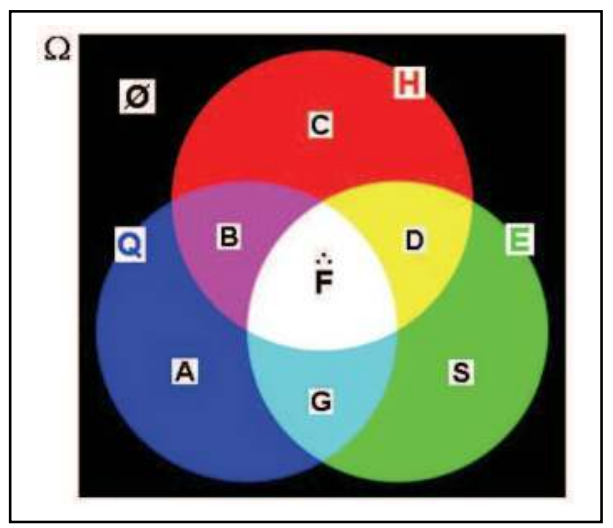

Fig. 3. Diagram showing the ecodesign model and the outcome subsets

The possible outcome sets and subsets can be expressed by the universal sample space as (Attaf, 2007)

$$
\Omega=\{\mathrm{Q}, \mathrm{H}, \mathrm{E}, A, B, C, D, S, G, \dot{F}, \varnothing\}
$$

in which,

$$
\begin{array}{lll}
A=Q \cap \bar{H} \cap \bar{E} & B=Q \cap H \cap \bar{E} & C=\bar{Q} \cap H \cap \bar{E} \\
D=\bar{Q} \cap H \cap E & S=\bar{Q} \cap \bar{H} \cap E & G=Q \cap \bar{H} \cap E \\
\therefore=Q \cap H \cap E & \varnothing=\bar{Q} \cap \bar{H} \cap \bar{E} &
\end{array}
$$

where, $\cap$ denotes intersection symbol, and $\bar{Q}, \bar{H}, \bar{E}$ are the complement of $Q, H, E$ and indicate that "Quality non-realizable", "Health non-realizable" and "Environment nonrealizable", respectively.

The subsequent analysis will concentrate only on the subset $\ddot{F}$, a unique searched subset that characterises the event: "intersection between $Q, H$ and $E$ does exist all the time".

\section{Application of probability principles to ecodesign function}

\subsection{Probability approach}

To illustrate the model-set probability, let us consider the sample space $\Omega$ that contains all the possible subsets (events) defined by Equation (1) and illustrated in Figure 3. Since the three key sets $Q, H$ and $E$ are composed of several variable elements associated to the different stages involved in the design process where each key set is assumed to fulfil a specific function; it can therefore be written that (Attaf, 2007):

$$
\begin{aligned}
Q & =\left\{x_{1}, x_{2}, x_{3}, \ldots . ., x_{m}\right\} \\
H & =\left\{y_{1}, y_{2}, y_{3}, \ldots . ., y_{n}\right\} \\
E & =\left\{z_{1}, z_{2}, z_{3}, \ldots . . ., z_{p}\right\}
\end{aligned}
$$

For better visualisation of different issues and calculation of the possible outcome probabilities, it is convenient to construct the probability tree diagram providing simple 
probabilistic measure. Figure 4 illustrates the different branches representing the possible events (Attaf, 2009).

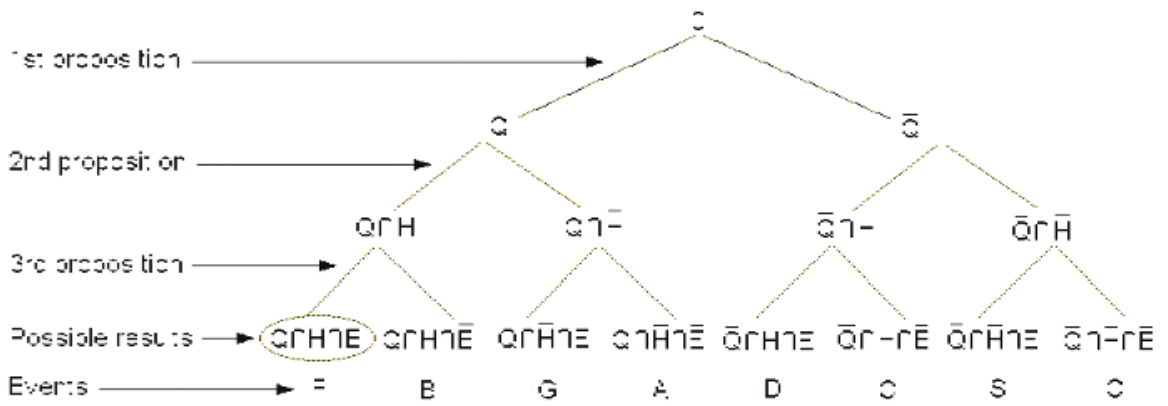

Fig. 4. Probability tree diagram and possible events

The probability theory will be applied to investigate the behaviour of the subset $\ddot{F}$, identified by the encircled area in Figure 4 . As a result, the corresponding event and its associated probability can respectively be written as (Attaf, 2007 \& 2009)

$$
\begin{gathered}
\dot{F}=Q \cap H \cap E \\
P(\dot{F})=P(Q \cap H \cap E)
\end{gathered}
$$

\subsection{Ecodesign function}

The probability $P(\ddot{F})$ represents the searched ecodesign function; a function that has multiple variables and lies between the values of 0 and 1 , and is defined by $f\left(Q=x_{i}, H=y_{i}, E=z_{i}\right)$. However, according to the nature of sets $Q, H$ and $E$ (independent or dependent) and the rules of multiplication in the probability theory, two possible cases (Figures $2 \mathrm{a}$ and $2 \mathrm{~b}$ ) may be presented; these are:

a. The sets $Q, H, E$ are independent (Figure 2a): a condition which does not satisfy the searched objective because the probability of the intersection is an empty set. Thus, it may be expressed by the following equation:

$$
f\left(Q=x_{i}, H=y_{i}, E=z_{i}\right)=P(\ddot{F})=P(\varnothing)=0
$$

b. The sets $Q, H, E$ are dependent (Figure $2 \mathrm{~b}$ and/or $2 \mathrm{c}$ ): a condition that does satisfy the searched objective. Thus, the probability of the occurred intersection can be written as:

$$
f\left(Q=x_{i}, H=y_{i}, E=z_{i}\right)=P(\ddot{F})=P(Q) \times P_{Q}(H) \times P_{Q \cap H}(E)
$$

where, $P(Q)$ represents the probability of an achievable quality;

$P_{Q}(H)$ represents the probability of an achievable health, knowing that quality has been achieved; and

$\mathrm{P}_{\mathrm{Q}_{\cap} \mathrm{H}}$ (E) represents the probability of an achievable environment, knowing that quality and health have been achieved. 
The following probability notations may be of some use in a certain literature reviews:

$$
\begin{gathered}
P_{Q}(H)=P(H \mid Q)=P(Q \cap H) / P(Q) \\
P_{Q \cap H}(E)=P(E \mid Q \cap H)=P(Q \cap H \cap E) / P(Q \cap H)
\end{gathered}
$$

Equations (7a) and (7b) are only valid when $P(Q)$ and $P(Q \cap H)$ are strictly greater than zero. On the other hand and according to the probability analysis, the realization of the event $\ddot{F}$ can take several values as a final result. This latter can be recapitulated by the following possible events (Attaf, 2007):

- If probability value is null $(P(\dot{\vec{F}})=0)$, then the event is impossible.

- If probability value is equal to $1(P(\ddot{F})=1)$, then the event is certain.

- If probability value is located between the two extreme values $(0<P(\ddot{F})<1)$, then it does exist a series of probable events.

\subsection{Practical descriptions of the variable probability elements}

To be familiar with the probability variable elements expressed by Equation (2), let us consider for example the statement "quality-assurance aspect is achievable and sustainable for the mould polymerisation process" and we let the letter " $x$ " denotes the property named "qualityassurance aspect is achievable and sustainable" and the subscript " $k$ " refers to the stage number involved in the design process, which corresponds here to "mould polymerisation process", we can then characterise the above statement as " $x_{k}$ ". For instance, if the subscript $k=4$, then the statement can be represented as " $x_{4}$ ". The circular limit line labelled " $Q$ " shown in Figure 3 encompasses the region that contains all the members that have the same property "qualityassurance aspect is achievable and sustainable" $\left(x_{1}, x_{2}, \ldots . ., x_{m}\right)$.

Proceeding in the same manner as previously, we may characterise the statement "environment-protection aspect is achievable and sustainable for resin type" as " $z_{k}$ ", where the letter " $z$ " denotes the property named "environment-protection aspect is achievable and sustainable" and the subscript " $k$ " denotes the stage number involved in the design process which corresponds here to "resin type". For instance, if the subscript $k=2$, then the previous statement will be symbolised as " $z_{2}$ ". The circular limit line called " $H$ " shown in Figure 3 encompasses the region that contains all the members that have the same property "environment-protection aspect is achievable and sustainable" $\left(z_{1}, z_{2}, \ldots ., z_{p}\right)$.

According to this representation for modelling, the property is always symbolised with a letter $x, y$ or $z$ associated to quality, health or environment, respectively. Whereas the stage number involved in the design process is symbolised with a subscript $k(k=1,2, \ldots, N)$. The statement is denoted by " $x_{k}$ ", " $y_{k}$ " or " $z_{k}$ ".

\section{Ecodesign coefficients}

\subsection{Identification of the eco-coefficients}

As there are $N$ successive stages in the design process, we found it convenient to assign to each of $Q, H$ and $E$ aspects a specific coefficient representing the probability of approval. When ecological considerations are taken into account, these coefficients are now called "eco-coefficients". From this standpoint, it may for stage $(k)$ be assumed that:

- $\quad \mathrm{a}=P\left(Q=x_{k}\right)$ is an eco-coefficient representing the probability of approval in terms of quality assurance, 
- $\quad \beta=P\left(H=y_{k} \mid Q=x_{k}\right)$ is an eco-coefficient representing the probability of approval with regard to health protection and known that quality is achieved; and

- $\quad \gamma=P\left(E=z_{k} \mid Q=x_{k} \cap H=y_{k}\right)$ is an eco-coefficient representing the probability of approval with regard to environmental preservation, known that health and quality are achieved. When the so-called "eco-coefficients" are taken into account during analysis and design of composite materials and structures, the terms "eco-analysis", "eco-formulation", "ecodesign", etc. can be considered to apply.

\subsection{Assessment of the eco-coefficients using code colours}

To further facilitate the understanding of the ecodesign modelling and the approach allowing the calculation of different eco-coefficients, we think that it will be easy to formulate the problem by simply referring to Figure 3, which illustrates the synthesis of additive colours between the three primary RGB ones. For instance, the red colour characterises the set $H$ (Health), the blue colour characterises the set $Q$ (Quality) and the green colour characterises the set $E$ (Environment). Moreover, combining these three primary colours in equal amount yields white that characterises the subset $\dot{F}$. Furthermore, superposing in equal amounts one of the primary colours with another colour gives a secondary colour; among them: yellow = red + green $($ subset $D)$, magenta $=$ red + blue $($ subset $B)$ and cyan $=$ blue + green $($ subset $G)$. The total absence of colours yields the black, an empty subset, symbolised by $\varnothing$.

The eco-coefficients $\alpha, \beta, \gamma$ may be located between $\alpha_{\mathrm{s}}$ and $1, \beta_{\mathrm{s}}$ and $1, \gamma_{\mathrm{s}}$ and 1 , respectively (i.e., $\left.\alpha_{s} \leq \alpha \leq 1 ; \beta_{s} \leq \beta \leq 1 ; \gamma_{s} \leq \gamma \leq 1\right)$. The subscript "s" refers to sustainability, whereas $\alpha_{s}, \beta_{s}$, $\gamma_{s}$ are sustainable coefficients, and their satisfaction measures are defined and established by sustainability requirements and eco-standards.

Table 1, shows in separate cases a rating satisfaction measure in the form of colour gauges when the events $Q, H$ and $E$ are independent and the values of $\alpha, \beta$ and $\gamma$ are varying separately.

\begin{tabular}{|c|c|c|c|c|}
\hline \multirow[b]{2}{*}{ Interval } & \multirow[b]{2}{*}{ Assessment } & \multicolumn{3}{|c|}{ Colour gauges } \\
\hline & & $\begin{array}{l}\text { Quality } \\
(q=\alpha)\end{array}$ & $\begin{array}{l}\text { Health } \\
(q=\beta)\end{array}$ & $\begin{array}{c}\text { Environment } \\
(q=\gamma)\end{array}$ \\
\hline $\mathrm{q}_{5} \leq \mathrm{q} \leq 1$ & Excellent & & & \\
\hline $\mathrm{q}_{4} \leq \mathrm{q}<\mathrm{q}_{5}$ & Very good & & & \\
\hline $\mathrm{q}_{3} \leq \mathrm{q}<\mathrm{q}_{4}$ & Good & & & \\
\hline $\mathrm{q}_{2} \leq \mathrm{q}<\mathrm{q}_{3}$ & Fair & & & \\
\hline $\mathrm{q}_{1} \leq \mathrm{q}<\mathrm{q}_{2}$ & Poor & & & \\
\hline $0 \leq \mathrm{q}<\mathrm{q}_{1}$ & Very poor & & & 0 \\
\hline
\end{tabular}

Table 1. Probability colour gauges for different values of $\alpha, \beta$ and $\gamma$ when $Q-H-E$ aspects are independent 
On the other hand, Table 2 illustrates an example in which the $Q-H-E$ aspects are dependent, $\alpha$ is fixed while $\beta$ and $\gamma$ are varying simultaneously.

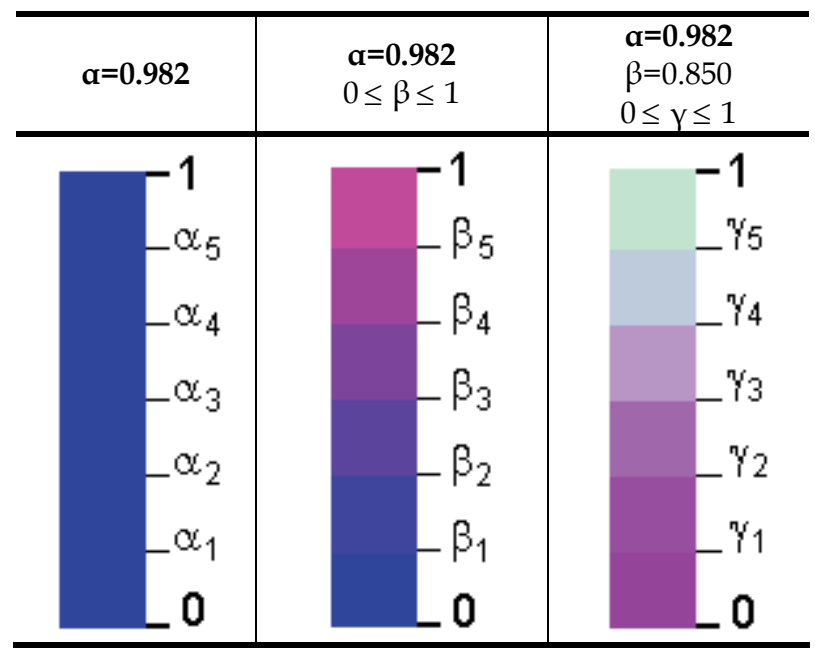

Table 2. Probability gauge for the eco-coefficients $\alpha, \beta$ and $\gamma$ when $Q-H-E$ aspects are dependent

From this primary analysis, it would be advantageous to exploit interesting results through the mixing additive colours technique when using the analogy between probability theory and code colours (Attaf, 2009). This technique can help designers and analysts involved with high-technology composite materials to easily understand the ecodesign approach and provide them with a strategic vision to reduce the negative impacts and assess the $Q-H-E$ performances during the composite product life-cycle.

\section{Ecodesign optimisation}

\subsection{Ecodesign flow-chart}

The objective is characterised by an optimisation of the ecodesign function, in which the $Q$ -

$H-E$ aspects all interact together (probability of the event $\ddot{F}$ ). The optimisation process is represented by a flow-chart and illustrated in Figure 6. The analysis of data after each loop for the stage $k=1$ will be iterated for the other stages involved in the design process $(k=2, \ldots, N)$. For instance, if the final probability output results are close to unity, then the objective previously outlined in Figure $2 \mathrm{c}$ is fully reached! However, if the output results are not close to the objective required by the ecodesign standards, we need to search for possible new alternatives. These alternatives can be provided by practical variation of the elements $x_{k}, y_{k}$ and $z_{k}$.

\subsection{Example of alternative solution}

To illustrate the procedure of optimisation, let us consider for example stage $k$ involved in the design process and let the subscript $k=4$ characterising for instance the sentence "openmould polymerisation process" for which we assume that the "quality assurance aspect" and the 


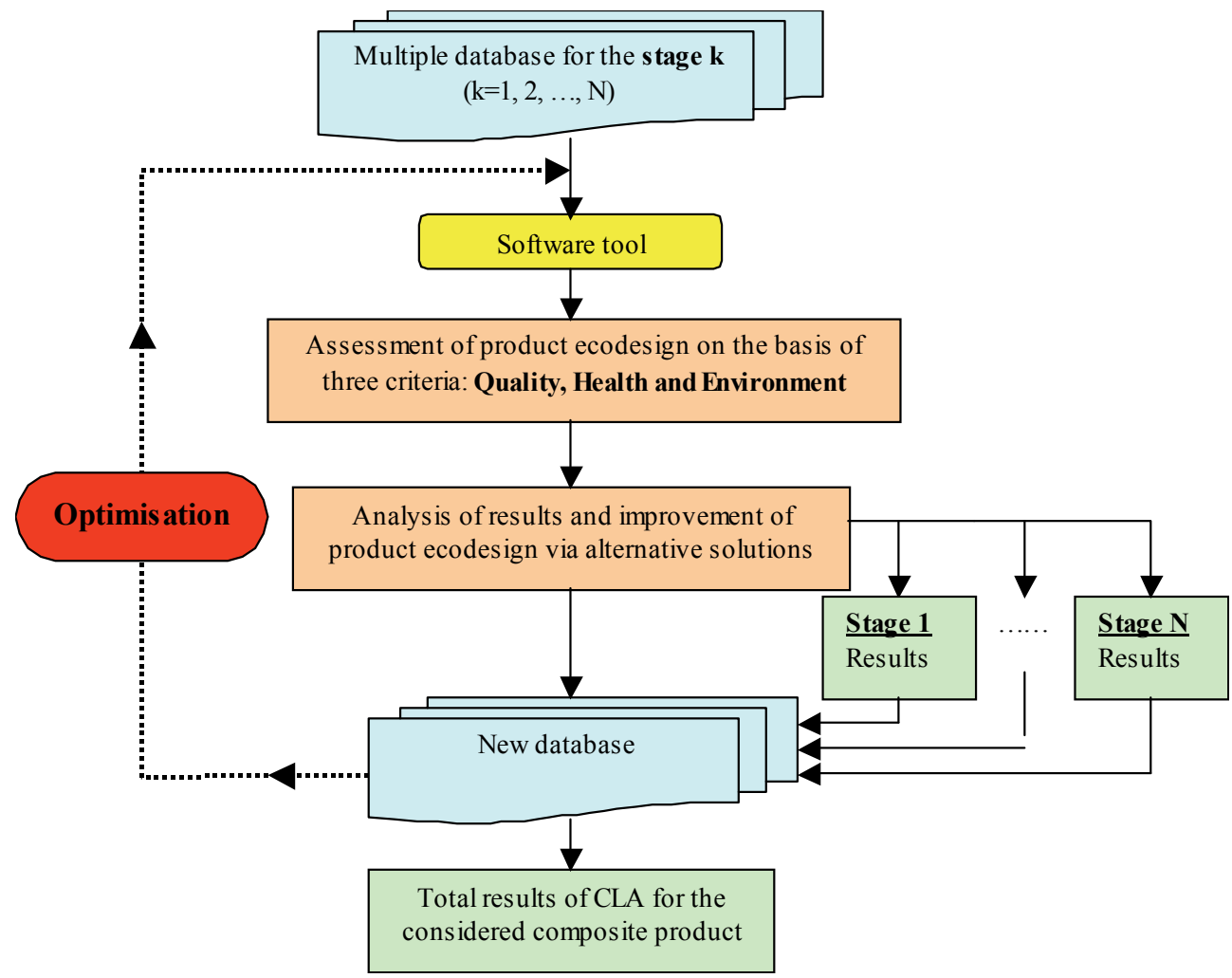

Fig. 5. Process flowchart for ecodesign of FRP composite product

"health protection aspect" symbolised respectively by " $x_{4}$ " and " $y_{4}$ " are achievable and sustainable; whereas the "environmental preservation aspect", symbolised by " $z$ " is unachievable for such a manufacturing process (open-mould). This means that the elements $x_{4}$ and $y_{4}$ belong simultaneously to both sets $Q$ and $H$; and therefore they belong to subset $B$ $\left(x_{4} \in B\right.$ and $\left.y_{4} \in B\right)$ as illustrated in Figure 6. However, the element $z_{4}$ does not belong to sets $Q$ and $H\left(z_{4} \notin Q\right.$ and $\left.z_{4} \notin H\right)$ but it belongs to set $E$; so it belongs to subset $S$. In order to realise the event $\ddot{F}$, the element $z_{4}$ must be altered in practice to become a new element satisfying the following statement "environmental preservation aspect is achievable and sustainable for closed-mould polymerisation process". With this alternative solution, achieved by a modification of the moulding process from open-mould to closed-mould, the three elements $x_{4}, y_{4}$ and $z_{4}$ related to stage 4 now belong to subset $\ddot{F}$ (Figure 6). Further alterations of these elements are possible in practice to reach the maximum limit target and to fully satisfy the ecodesign requirements.

As discussed previously, if we let " $z_{1}$ " denotes the statement "environmental preservation aspect is unachievable for polyester resin with high-styrene-content", this can be practically changed to yield a new element satisfying the statement "environmental preservation aspect is achievable for polyester resin with low-styrene-content". By replacing "high-styrene-content" with "low-styrene-content", this gives a new " $z_{1}$ " that satisfies the ecodesign requirements. 


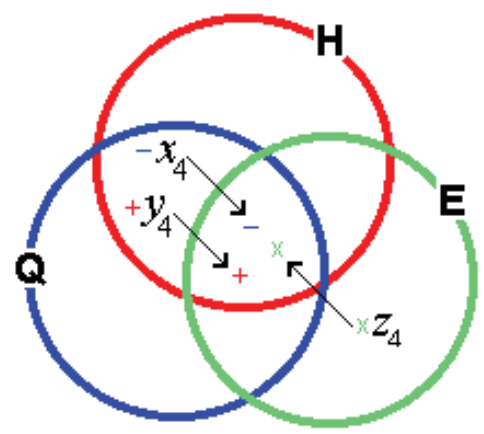

Fig. 6. Example of alternative solution related to stage 4

\subsection{Multidimensional optimisation software tool}

Given the complexity of the optimisation process, which has to be applied in each phase involved in the design of a FRP composite product whilst taking into account the three key criteria $Q, H \& E$, it is important to develop a software tool capable to solve the probability problem and provide a high technique that can optimise the ecodesign function.

In this software tool, the optimisation process is composed of three steps which are recapitulated as follows: the first step defines the stage involved in the design process $(k=1,2, .$.$) , collects the associated data and specifies the target value of probability to be$ reached; the second step assesses and analyses results on the basis of the three main criteria $(Q, H \& E)$ and then applies if necessary the optimisation procedure to achieve the required value of probability; and the third step reports in the form of a specification sheet the final eco-results and provides a comparison with classical ones.

The analysis of the ecodesign function will be made systematically by phase and according to the type of data collected, the assessment may be done using qualitative and/or quantitative method. Among the main phases subjected to an assessment procedure for FRP composite products are included:

- $\quad$ Phase 1: Definition of the FRP composite product functions and the design objectives.

- $\quad$ Phase 2: Choice of the constituents and the manufacturing processes

- $\quad$ Phase 3: Characterisation of the different materials and definition of thicknesses

- $\quad$ Phase 4: Correlation between tests and calculations

- $\quad$ Phase 5: Manufacture and inspection of the first FRP composite article (FAI)

- $\quad$ Phase 6: Qualification and certification of the FRP composite product

- $\quad$ Phase 7: Launch of the FRP composite product manufacturing on the Supply Chain

- $\quad$ Phase 8: Marketing of the FRP composite product (distribution and transport)

- $\quad$ Phase 9: Use, repair and maintenance of the FRP composite product

- $\quad$ Phase 10: End of life of the FRP composite product, waste management and recycling

- $\quad$ Phase 11: Evolution of lifecycle FRP composite product

For better interpretation and visualisation of results, the global ecodesign assessment of the FRP composite product may be given in the form of a spider graph showing the location of eco-coefficients values with regards to the limiting envelop of the ecodesign function. In addition, the other constraints such as: hygiene \& security, risk, energy, cost, time factor, etc.. may be included in one of the three aspects that best matches the study. 


\section{Material eco-characterisation and advanced eco-formulations}

Throughout the design process, it is considered that the condition of intersection between $Q$, $H \& E$ is always performed, so the product of the associated eco-coefficients. For notation simplicity, this product expressed by $\alpha \times \beta \times \gamma$ may be reduced and replaced by a single factor called "eco-efficiency factor", denoted for example by the Greek letter $\lambda$. This factor will be inserted into each step involved in the design process and its measured value may be different from one stage to another. For the stage $(k)$, the ecodesign performance is evaluated in relation to the measurement results of the factor $\lambda(k)$ equals to $\alpha^{(k)} \times \beta^{(k)} \times \gamma^{(k)}$. For instance, if this factor reaches the sustainable factor i.e., $\lambda(k)=\lambda_{s}^{(k)}$, the objective required is then attained and the ecodesign condition is fully accomplished for that stage. However, if the factor $\lambda(k)$ fails in stage $(k)$, this will cause a systematic rejection of the material, the process, the method or the service used in that stage. An alternative solution is absolutely necessary before performing the next stage $(k+1)$.

\subsection{Eco-characterisation of composite materials}

To further explain this approach, let us consider a simple standard material characterisation test under uniaxial mechanical tension, chosen because it is the easiest and most convenient mechanical test (Attaf, 2008). For better understanding and more explicit interpretation, the results are presented in the form of conventional stress-strain diagram, where the $x$-axis represents strain $\varepsilon$ and the $y$-axis represents stress $\sigma$, but to clearly illustrate the different successive stages of material behaviour, we consider a ductile elastic-plastic material. As a result, the stress-strain curve in Figure 7 illustrates the linear and non-linear elastic domains, the plastic domain, and the corresponding failure point. However, no information on the material behaviour with respect to the environment and health protection is given. Since our approach is essentially based on this argument, we consider that the absence of such information in the final results may cause serious problems for human health and the environment in the short or long term (e.g., asbestos). To overcome these inconveniences and achieve ecodesign approach, it is imperative to consider the environmental and health constraints in more detail and integrate them into the output results of test.

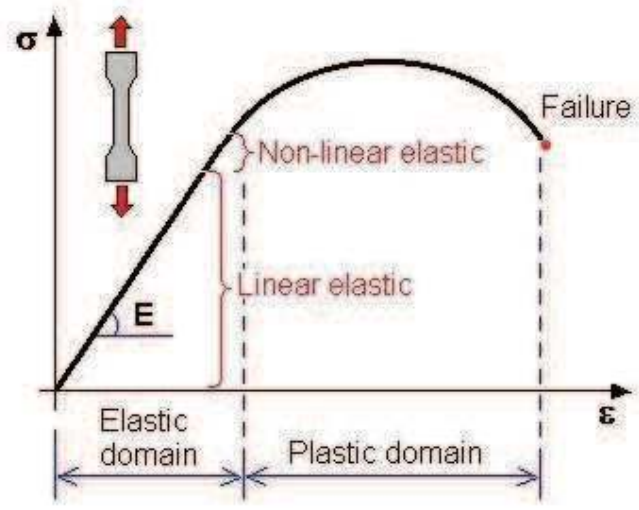

Fig. 7. Stress-strain relationship with no information on the material behaviour in relation to health and environmental aspects 
This approach allows deciding very early on in the material selection process whether to approve or reject a proposed material.

In the stage of material characterisation, the environmental and health impacts are taken into account by considering the eco-efficiency factor $\lambda$. This factor will be inserted into the standard mechanical characterisation formulae of materials. Hence, Young's modulus of elasticity, determined by Hooke's law and derived from experimental results must be adapted to the actual situation by integrating health and environmental considerations into characterisation tests. Thus, the new Young's modulus of elasticity will become $\ddot{E}$ "Young's eco-modulus ". The three dots $(\therefore)$ above the character $E$ are only a brief description of the interaction between $Q, H \& E$ as discussed previously in Section 2.2.

In relation to this orientation, the modulus eco-efficiency may be evaluated by measuring the elasticity eco-efficiency factor, defined by the following ratio (Attaf, 2008):

$$
\frac{\ddot{E}}{E}=\lambda
$$

The numerator value of $\ddot{E}$ approaches the denominator value $E \neq 0$, when $\lambda$ tends towards unity. As it was discussed earlier, if this value does not meet ecodesign requirements, a search for possible new alternatives can be provided to maximise the eco-coefficients until sufficient agreement is achieved. Further investigations on the optimisation of these ecocoefficients are still necessary, however.

\subsection{Generalized Hooke's eco-law for unidirectional ply}

In high-performance composite structures, structural components are manufactured mainly from unidirectional fibre-reinforced plies, where the ply axes are identified in the principal coordinate system with numbers 1,2 and 3. Whereas, the laminate axes are identified in the global coordinate system (Cartesian coordinates) with letters $x, y$ and $z$ (Jones, $1999 \&$ Saarela, 1994). The 1-axis and $z$-axis are combined into a single axis (Figure 8).

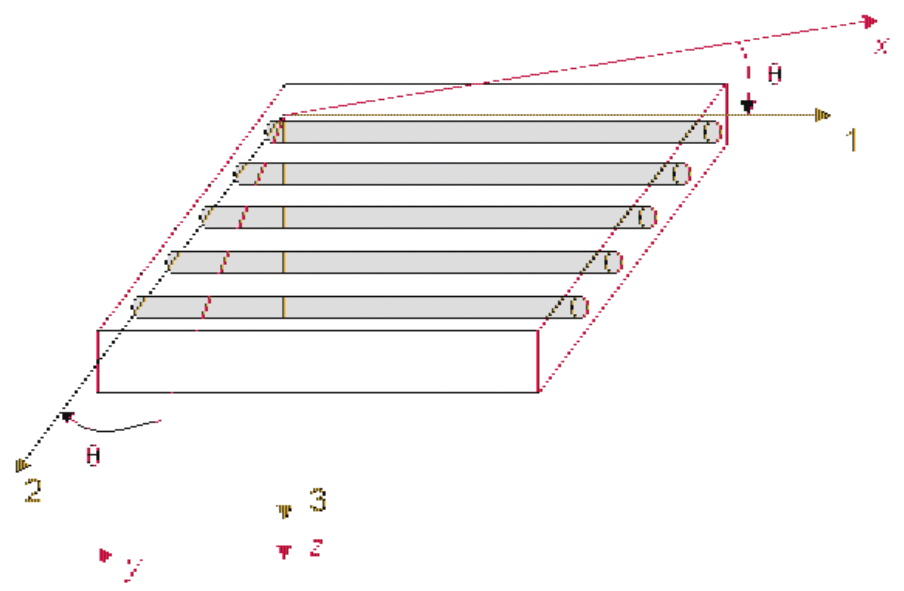

Fig. 8. Principal coordinate system $(1,2,3)$ vs. global coordinate system $(x, y, z)$ for unidirectional ply 
The Young's moduli $E_{1}, E_{2}, E_{3}$ and the shear moduli $G_{12}, G_{23}, G_{13}$ of the unidirectional ply determined from experimental tests will become $\ddot{E}_{1}, \dot{\ddot{E}}_{2}, \quad \ddot{E}_{3}, \quad \ddot{G}_{12}, \quad \ddot{G}_{23}, \quad \ddot{G}_{13}$, respectively. In relation to this orientation and for a unidirectional fibre-reinforced ply, the eco-efficiency factors related to the engineering constants for the linear-elastic mechanical behaviour may be defined in the principal coordinate system as (Attaf, 2008):

$$
\lambda_{i}=\frac{\ddot{E}_{i}}{E_{i}}, \quad \lambda_{i j}=\frac{\dot{G}_{i j}}{G_{i j}} \quad \text { with } \frac{\dot{E_{i}}}{v_{i j}}=\frac{\ddot{E}_{j}}{v_{j i}} \quad(i, j=1,2,3 \text { and } i \neq j)
$$

where $E_{i}, \ddot{E}_{i}$ are classical and sustainable Young's moduli in $i$-direction (i=1, 2 and 3),

$G_{i j}, \dot{G}_{i j}$ are classical and sustainable shear moduli in the $i-j$ plane $(i-j=2-3,3-1$ and 1-2),

$\lambda_{i}, \lambda_{i j}$ are the eco-efficiency factors of Young's and shear moduli, respectively and,

$v_{i j}$ is the Poisson's ratio for transverse strain in the $j$-direction when stressed in the $i$ direction. For this value, it is important to note that no attempt was made to investigate the sustainability of Poisson's ratios. Their influence is beyond the scope of this analysis.

According to these assumptions and generalized Hooke's law, the strain-stress eco-relations for an orthotropic material in the principal coordinate system $(1,2,3)$ may be written in compact matrix form as:

$$
\{\varepsilon\}_{123}=\left[\dot{S_{i j}}\right]_{123}\{\sigma\}_{123} \quad(i, j=1,2, . ., 6)
$$

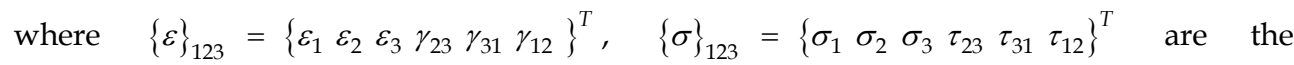
transpose of the strain and stress vectors, and $\left[\dot{S_{i j}}\right]_{123}$ is the eco-compliance matrix of order $6 \times 6$ (6 rows by 6 columns). The components $\dot{S}_{i j}=\dot{S}_{j i}$ are defined as:

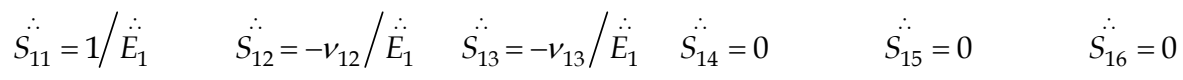

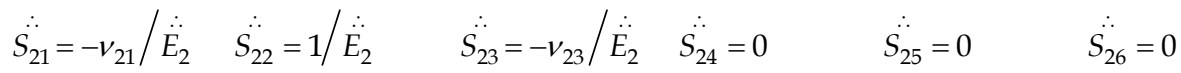

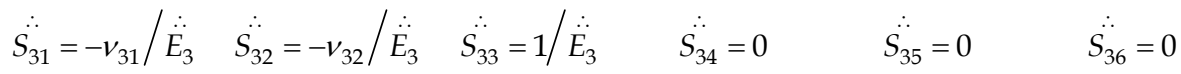

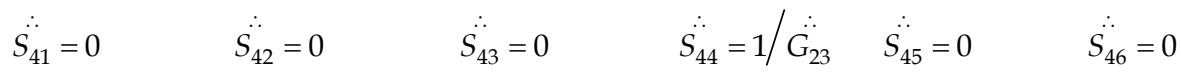

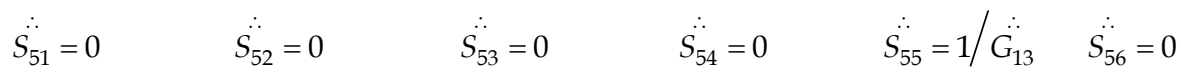

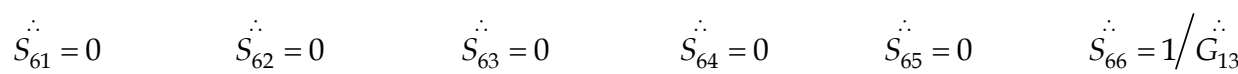

Also, the eco-stiffness matrix of the ply may be obtained by the inverse of the ecocompliance matrix. Thus, we can write: 


$$
\left[\therefore Q_{i j}\right]_{123}=\left[\therefore \dot{S}_{i j}\right]_{123}^{-1} \quad(i, j=1,2, . ., 6)
$$

When using the eco-stiffness matrix, the stress-strain relations can be obtained by the inverse of Equation (10). Thus, we obtain:

$$
\{\sigma\}_{123}=\left[\therefore Q_{i j}\right]_{123}\{\varepsilon\}_{123} \quad(i, j=1,2, . ., 6)
$$

Since orthotropic fibres have almost the same characteristics along 2- and 3-axis, the 2-3 plane is considered to be a plane of symmetry and the three-dimensional 123-coordinate system will be reduced to two-dimensional 12-coordinate system. Thus, Equation (12a) becomes:

$$
\{\sigma\}_{12}=\left[\therefore Q_{i j}\right]_{12}\{\varepsilon\}_{12} \quad(i, j=1,2,6)
$$

where $\{\sigma\}_{12}=\left\{\begin{array}{lll}\sigma_{1} & \sigma_{2} & \tau_{12}\end{array}\right\}^{T},\{\varepsilon\}_{12}=\left\{\begin{array}{lll}\varepsilon_{1} & \varepsilon_{2} & \gamma_{12}\end{array}\right\}^{T}$ are the stress and strain vectors in 12coordinate system, and $\left[\begin{array}{c}\therefore \\ Q_{i j}\end{array}\right]_{12}$ is the eco-stiffness matrix of order $3 \times 3$ (3 rows by 3 columns).

Using the transformation matrix, Equation (12b) may be written, after some rearrangements, in the global $x y$-coordinate system as:

$$
\{\sigma\}_{x, y}=\left[\begin{array}{c}
\therefore \\
Q_{i j}
\end{array}\right]_{x, y}\{\varepsilon\}_{x, y} \quad(i, j=1,2,6)
$$

where $\{\sigma\}_{x, y}=\left\{\sigma_{x} \sigma_{y} \tau_{x y}\right\}^{T},\{\varepsilon\}_{x, y}=\left\{\begin{array}{lll}\varepsilon_{x} & \varepsilon_{y} & \gamma_{x y}\end{array}\right\}^{T}$ are the stress and strain vector in the global $x y$-coordinate system, and $\left[\begin{array}{c}\bar{\therefore} \\ Q_{i j}\end{array}\right]_{x, y}$ is the eco-stiffness matrix of order $3 \times 3$ (3 rows

$$
\therefore \quad \bar{C}
$$

by 3 columns). The components $Q_{i j}=Q_{j i}(i, j=1,2,6)$ are functions of the fibre orientation angle, $\theta$ and the orthotropic elastic eco-moduli of the ply. By letting $C=\cos \theta$ and $S=\sin \theta$, the eco-stiffness components are defined in the global $x y$-coordinate system as:

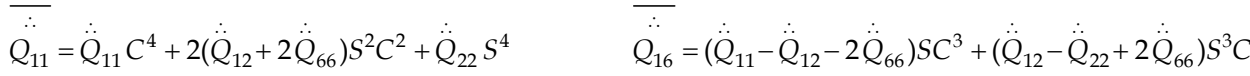

$$
\begin{aligned}
& \bar{\therefore}=\left(\ddot{Q}_{11}+\ddot{Q}_{22}-4 \ddot{Q}_{66}\right) S^{2} C^{2}+\ddot{Q}_{12}\left(S^{4}+C^{4}\right) \quad \bar{\therefore}=\left(\ddot{Q}_{11}-\ddot{Q}_{12}-2 \ddot{Q}_{66}\right) S^{3} C+\left(\ddot{Q}_{12}-\ddot{Q}_{22}+2 \ddot{Q}_{66}\right) S C^{3} \\
& \therefore \quad \therefore \ddot{Q}_{22}=\dot{Q}_{11} S^{4}+2\left(\ddot{Q}_{12}+2 \ddot{Q}_{66}\right) S^{2} C^{2}+\ddot{Q}_{22} C^{4} \quad \therefore \quad \therefore_{66}=\left(\ddot{Q}_{11}+\ddot{Q}_{22}-2 \ddot{Q}_{12}-2 \ddot{Q}_{66}\right) S^{2} C^{2}+\ddot{\mathscr{Q}}_{66}\left(S^{4}+C^{4}\right)
\end{aligned}
$$


With these ecological considerations, the constituent equations for laminated composite structures can be derived using the classical lamination theory (Jones, 1999).

\subsection{Constitutive eco-equations of laminates}

When the environment and health impacts besides quality are taken into consideration in the structural analysis, the constitutive relations for an unsymmetrically $n$-layered laminated composite plate $(k=1,2, . . n)$, and without transverse shear deformations can, after integration through each ply thickness and summation, be written in matrix form as:

$$
\left\{\begin{array}{l}
N_{x} \\
N_{y} \\
N_{x y} \\
M_{x} \\
M_{y} \\
M_{x y}
\end{array}\right\}=\sum_{k=1}^{n} \int_{z_{k-1}}^{z_{k}}\left\{\begin{array}{c}
\sigma_{x} \\
\sigma_{y} \\
\tau_{x y} \\
z \sigma_{x} \\
z \sigma_{y} \\
z \tau_{x y}
\end{array}\right\} d z=\left[\begin{array}{cccccc}
\ddot{A}_{11} & \ddot{A}_{12} & \ddot{A}_{16} & \dot{B}_{11} & \dot{B}_{12} & \ddot{B}_{16} \\
& \ddot{A}_{22} & \ddot{A}_{26} & \ddot{B}_{12} & \ddot{B}_{22} & \ddot{B}_{26} \\
& & \dot{A}_{66} & \ddot{B}_{16} & \ddot{B}_{26} & \ddot{B}_{66} \\
& & & \ddot{D}_{11} & \ddot{D}_{12} & \ddot{D}_{16} \\
& & & & \dot{D}_{22} & \dot{D}_{26} \\
& s y m & & & & \ddot{D}_{66}
\end{array}\right]\left\{\begin{array}{l}
\varepsilon_{0 x} \\
\\
\\
\end{array}\right.
$$

Or in compacted matrix form:

$$
\left\{\frac{N}{M}\right\}_{x y}=\sum_{k=1}^{n} \int_{z_{k-1}}^{z_{k}}\left\{\frac{\{\sigma\}_{x y}}{z z\{\sigma\}_{x y}}\right\} d z=\left[\begin{array}{c|c}
\ddot{A} & \dot{B} \\
\hline \dot{B} & \dot{\ddot{D}}
\end{array}\right]\left\{\begin{array}{l}
\varepsilon^{0} \\
\hdashline \kappa
\end{array}\right\}_{x y}
$$

where $N$ and $M$ are the resultant in-plane forces and bending/torsional moments, respectively. Whereas, $\varepsilon^{0}$ and $\kappa$ are the associated strains and curvatures.

The eco-components of the sub-matrices $\ddot{A}_{i j}$ (extensional eco-stiffnesses), $\ddot{B}_{i j}$ (coupling ecostiffnesses) and $\ddot{D}_{i j}$ (bending eco-stiffnesses) are expressed as:

$$
\left(\dot{A_{i j}}, \dot{B_{i j}}, \dot{\mathscr{D}_{i j}}\right)=\sum_{k=1}^{n} \int_{z k-1}^{z k}\left(\overline{\ddot{Q}}_{i j}\right)_{k}\left(1, z, z^{2}\right) d z \quad(i, j=1,2,6)
$$

These sub-matrices may be assembled in a single matrix $[\dot{C}]$ called extensional-couplingbending eco-stiffness matrix. Thus,

$$
[\ddot{C}]=\left[\begin{array}{c|c}
\ddot{A} & \ddot{B} \\
\hdashline \ddot{B} & \ddot{D}
\end{array}\right]
$$

and Equation (14b) may be written as:

$$
\left\{\frac{N}{M}\right\}_{x y}=[\ddot{C}]\left\{\begin{array}{l}
\varepsilon^{0} \\
\bar{\kappa}
\end{array}\right\}_{x y}
$$




\subsection{Finite element eco-approach}

When the analytical or mathematical solution does not exist, numerical methods such as finite element method (Jones, 1999 and Zienkiewicz et al, 2005) may be used to yield an approached solution. However, to achieve sustainable structural performance that is also based on an assessment of environmental and health performances, it is imperative to consider these constraints in more detail and integrate them into the finite element analysis. In such situation, the finite element eco-approach for dynamic analysis will first consist in resolving for one element type $(e)$ the system of the following equations:

$$
\left[M_{e}\right]\left\{\ddot{q}_{e}(t)\right\}+\left[\dot{k}_{e}\right]\left\{q_{e}(t)\right\}=\left\{f_{e}(t)\right\}
$$

where $\left\{q_{e}(t)\right\}$ is the vector of nodal displacements and rotations, $\left\{\ddot{q}_{e}(t)\right\}$ is the vector of accelerations, and $\left\{f_{e}(t)\right\}$ is the vector of forcing functions (external loads). Whereas $\left[M_{e}\right]$ and $\left[\ddot{k}_{e}\right]$ are the mass matrix and eco-stiffness matrix, respectively. These are defined by the following expressions:

$$
\begin{gathered}
{\left[M_{e}\right]=\int_{\Omega_{e}}[E]^{T}[\bar{m}][E] d \Omega_{e}} \\
\dot{\left.\dot{k}_{e}\right]}=\int_{\Omega_{e}}[S]^{T}[\ddot{C}][S] d \Omega_{e}
\end{gathered}
$$

in which $\Omega_{e}$ is the surface of an element; $[\bar{m}]$ is the inertia matrix, $[E]$ is the displacement function matrix, $[S]$ is the strain interpolation matrix, and $[\ddot{C}]$ is the elastic extensionalcoupling-bending eco-stiffness matrix. The Jacobian matrix $[J]$ is used to allow the passage from Cartesian coordinates $(x, y, z)$ to natural coordinates $(\xi, \eta, \zeta)$.

For static analysis, the acceleration vector vanishes and Equation (18) is independent of time and becomes as follows:

$$
\left[\dot{k}_{e}\right]\left\{q_{e}\right\}=\left\{f_{e}\right\}
$$

Assembling eco-stiffness and mass matrices, and forcing functions vector of each element involved into the generation of the whole structure yields the global system of equations:

- For dynamic eco-analysis: $\quad[M]\{\ddot{q}(t)\}+[\ddot{K}]\{q(t)\}=\{F(t)\}$

- For static eco-analysis:

$$
[\ddot{K}]\{q\}=\{F\}
$$

where $\{q\}$ is the vector of global displacements, $\{\ddot{q}\}$ is the vector of global accelerations, $\{F\}$ is the vector of external loads (vanishes for free vibration analysis), $[M]$ is the global mass matrix, and $[\ddot{K}]$ is the global eco-stiffness matrix.

By comparing the eco-results with the classical ones that do not take into account environmental and health considerations, we can yield an estimate difference value called "eco-deviation", which may be calculated using the following relation: 


$$
\text { Eco-deviation }(\%)=\left|\frac{\dot{V}-V}{V}\right| \times 100
$$

where $\ddot{V}$ is the eco-result corresponding to $\lambda=\lambda_{i}\left(0<\lambda_{i}<1\right)$ and $V$ is the classical result corresponding to $\lambda=1$, generally.

For better comment and understanding of this eco-approach, the final results may be presented in the form of graphs with normalized axes. A dimensionless quantity named "eco-efficiency ratio", representing the ratio between eco-results and classical ones, can be chosen for the $y$-axis and denoted for example by the Greek letter $\Lambda=\ddot{V} / V$. When performing stress analysis of composite materials and structures, $\Lambda$ can for instance be equal to $\dot{\sigma} / \sigma$ or $\dot{\tau} / \tau$. The discrepancy between classical and sustainable results can help designers and analysts to evaluate easily environmental and health performances. Moreover, this discrepancy can be minimised via alternative solutions to reach the appropriate value required by ecodesign standards.

\section{Conclusion}

Based on mathematical formulations, scientific, industrial and technological know-how in the field of FRP composite materials and structures, this contribution aims to innovate and develop a new approach providing the integration at each stage of the designing process three balanced key criteria characterised mainly by Quality assurance, Health protection and Environmental preservation $(Q, H \& E)$. To achieve these requirements, new criteria in the form of eco-coefficients were defined and developed. However, greater depth of study is still required to establish the rating satisfaction measure that yields the appropriate values of sustainable coefficients, which are considered as an important source of reference for comparison survey. To approach sustainability values of these coefficients, probability analysis of ecodesign function and some optimisation procedures based on a new technique of additive colours were undertaken in accordance with the three balanced key criteria. When these eco-coefficients are approved by sustainability standards, they can then be integrated into formulations of design and analysis, in characterisation tests; they can also be implemented into future finite-element computer programs, etc. Designers, analysts and engineers can make better use of ecodesign aspects to assess environmental and health performances when comparing eco-results with classical ones.

This investigation could be integrated in the international standards, codes and guidelines for sustainability research actions, and contribute to new orientations in the design of ecofriendly composite materials and structures. It may also be regarded as a stimulation of ecoinnovation, sustainability and research activities in the field of FRP composite products ecodesign and as an encouragement for designers and engineers to have a great motivation towards the integration of health and environmental aspects into the designing process. In addition, new ecodesign recommendations could be developed via this innovative survey. These recommendations will, however, increase the design space of future composite materials and their products. This will offer a new data to use in evaluating the different stages of a material/process/product life-cycle.

This type of "eco-action" constitutes a multidisciplinary approach that can involve specialists in mechanical/civil/structural and process engineering, mathematics, physics, 
chemistry, health, environment and sociology. In addition, the impacts that may be investigated including: (1) the undesirable substances entering in the manufacturing process, (2) the amount of emissions of greenhouse gases, (3) the level of $Q-H-E$ interaction, (4) the quantity of the waste production and expired materials, (5) the classification of the company with regard to the authorized regulations, etc. Then, alternative solutions leading to new methods of ecodesign are suggested. The approved eco-coefficients may become a source of normative coefficients used for validating the different manufacturing stages and qualifying \& certifying the new developed eco-composite materials and structures.

\section{References}

Attaf, B. \& Hollaway, L. (1990a). Vibrational analyses of stiffened and unstiffened composite plates subjected to in-plane loads. Composites Vol.21, No.2, (March 1990) pp.117-126, ISNN 0010-4361

Attaf, B. \& Hollaway, L. (1990b). Vibrational analyses of glass reinforced polyester composite plates reinforced by a minimum mass central stiffener. Composites Vol.21, No.5, (Sept.1990) pp.425-430, ISNN 0010-4361

Attaf, B. (2007). Towards the optimisation of the ecodesign function for composites. JEC Composites. No.34, (July-August 2007) pp.58-60, ISNN 1639-965X

Attaf, B. (2008). Eco-characterisation of composite materials. JEC Composites. No.42, (JulyAugust 2008) pp.58-60, ISNN 1639-965X

Attaf, B. (2009). Probability approach in ecodesign of fibre-reinforced composite structures. Proceedings of CAM'2009 Conference, Algeria, November 2009, Biskra

Hollaway, L. \& Attaf, B. (1989). On the vibration of glass/polyester composite stiffened and unstiffened rectangular plates. Proceedings of the Seventh Int. Conf. on Composite Materials, pp. 435-444, China, October 1989, Chinese Society of Aeronautics, Beijing

Jones, R.M. (1999). Mechanics of composite materials. Taylor \& Francis, Inc., ISBN 1-56032-712$X$, Philadelphia

Saarela, O. (1994). Computer programs for mechanical analysis and design of polymer matrix composites. Prog. Polym. Sci. Vol.19, pp 58-60, ISNN 0079-6700

Yang, T.Y. (1986). Finite element structural analysis. Prentice-Hall, Inc., ISBN 0-13-317116-7, New Jersey

Zienkiewicz, O.C., Taylor, R.L. \& Zhu, J.Z. (2005). The finite element method: its bases and fundamentals. Elsevier Butterworth-Heinemann, ISBN 075066320 0, Oxford 


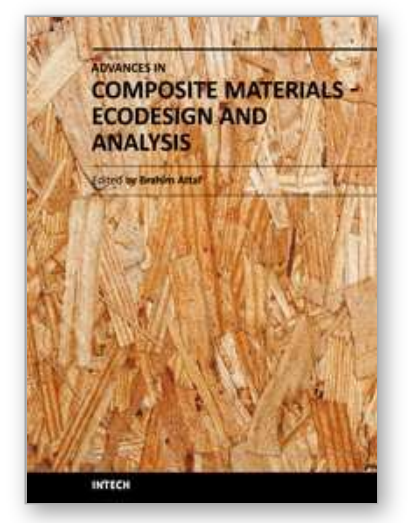

\author{
Advances in Composite Materials - Ecodesign and Analysis \\ Edited by Dr. Brahim Attaf
}

ISBN 978-953-307-150-3

Hard cover, 642 pages

Publisher InTech

Published online 16, March, 2011

Published in print edition March, 2011

By adopting the principles of sustainable design and cleaner production, this important book opens a new challenge in the world of composite materials and explores the achieved advancements of specialists in their respective areas of research and innovation. Contributions coming from both spaces of academia and industry were so diversified that the 28 chapters composing the book have been grouped into the following main parts: sustainable materials and ecodesign aspects, composite materials and curing processes, modelling and testing, strength of adhesive joints, characterization and thermal behaviour, all of which provides an invaluable overview of this fascinating subject area. Results achieved from theoretical, numerical and experimental investigations can help designers, manufacturers and suppliers involved with high-tech composite materials to boost competitiveness and innovation productivity.

\title{
How to reference
}

In order to correctly reference this scholarly work, feel free to copy and paste the following:

Brahim Attaf (2011). Generation of New Eco-friendly Composite Materials via the Integration of Ecodesign Coefficients, Advances in Composite Materials - Ecodesign and Analysis, Dr. Brahim Attaf (Ed.), ISBN: 978953-307-150-3, InTech, Available from: http://www.intechopen.com/books/advances-in-composite-materialsecodesign-and-analysis/generation-of-new-eco-friendly-composite-materials-via-the-integration-of-ecodesigncoefficients

\section{INTECH}

open science | open minds

\section{InTech Europe}

University Campus STeP Ri

Slavka Krautzeka 83/A

51000 Rijeka, Croatia

Phone: +385 (51) 770447

Fax: +385 (51) 686166

www.intechopen.com

\section{InTech China}

Unit 405, Office Block, Hotel Equatorial Shanghai

No.65, Yan An Road (West), Shanghai, 200040, China 中国上海市延安西路65号上海国际贵都大饭店办公楼 405 单元

Phone: +86-21-62489820

Fax: $+86-21-62489821$ 
(C) 2011 The Author(s). Licensee IntechOpen. This chapter is distributed under the terms of the Creative Commons Attribution-NonCommercialShareAlike-3.0 License, which permits use, distribution and reproduction for non-commercial purposes, provided the original is properly cited and derivative works building on this content are distributed under the same license. 